This is an Accepted Manuscript of an article published in Disability \& Society on 4 July 2014, available online: http://www.tandfonline.com/10.1080/09687599.2014.923750.

Anne Lindblom, Department of Educational Studies, Karlstad University, Sweden. School of Educational Sciences and Psychology, University of Eastern Finland, Joensuu, Finland.

\title{
Under-detection of autism among First Nations children in British Columbia, Canada.
}

\begin{abstract}
This article shows that First Nations children diagnosed with autism in British Columbia (BC) Canada are underrepresented in publications regarding autism and the prevalence thereof and that this group appears to be under-detected. The aim of this review of publications regarding autism and aboriginal populations in Canada and other countries is to examine possible explanations. The research review results suggest that possible reasons for under-detection of autism among aboriginal populations, and consequently First Nations peoples, can be diagnostic substitution and symptom presentation, ethnic or cultural, area of residence or the impact of historical oppression and discrimination.
\end{abstract}

Keywords: autism, First Nations, aboriginal, prevalence, under-detection, British Columbia, Canada.

\section{Introduction}

In May 2013 the Fifth Edition of the Diagnostic and Statistical Manual of Mental Disorders (DSM-5) was released and important changes were made in the criteria for autism spectrum disorder (ASD), herein also referred to as autism. There are no longer sub-diagnoses and the diagnostic criteria are grouped in two areas, social communication and interaction and restricted and repetitive behaviors (Hyman, 2013). The prevalence of autism spectrum disorders seems to be increasing (Biao, 2012; Matson \& Kozlowski, 2011; National Epidemiologic Database for the Study of Autism in Canada, NEDSAC, 2012).

It can be seen as DSM-5 is based on a western, medical, deficit model which may not be fully applicable to aboriginal peoples. Autism may on the other hand be considered as a social construct. Psychiatric diagnoses are in Szasz (1974) view, stigmatizing labels. Foucault (1988) raises issues of power and exclusion in the social construction of mental illness. Stienstra (2012) argues that race/ethnicity, heritage language and religion are factors that should be regarded in disability studies, in Canada specifically, but of course in all disability research. 
This is an Accepted Manuscript of an article published in Disability \& Society on 4 July 2014, available online: http://www.tandfonline.com/10.1080/09687599.2014.923750.

Anne Lindblom, Department of Educational Studies, Karlstad University, Sweden. School of Educational Sciences and Psychology, University of Eastern Finland, Joensuu, Finland.

Three groups of aboriginal people are recognized by the Canadian constitution. Indians (First Nations), Métis and Inuit are descendants of the original peoples of North America (AANDC, 2012a). An Indian person can be either Status or Non-status depending on if they are registered as an Indian under the Indian Act or not (AANDC, 2012b). If a person has Indian status they are recognized and registered as an Indian by the federal government. A Status Indian is eligible for services and programs from the provincial and federal government (AANDC, 2012c). The Indian Act contains obligations of the federal government and regulates management of Indian resources such as reserve lands and money. It was first passed in 1876 but changes have been made several times since then for example regarding who is eligible for status (AANDC, 2012b). According to AANDC (2013a) there are over one million people that identify themselves as aboriginal and in the 617 First Nations communities there are more than 50 nations or cultural groups. 53\% of the aboriginal population in Canada are Status and 11\% are Non-status Indians. First Nations or Indian bands are usually located on reserve lands (AANDC, 2012a) and individuals can live either on or off reserve. In this article the term First Nations means both Status and Non-status Indians.

It may seem like the native population of Canada has many rights but there is a complex set of regulations that determine what applies to each individual. In 2006 there were 134180 North American Indians in British Columbia, BC. Compared to non-aboriginals, people within the First Nations population in BC have a lower level of education. For instance in 2006, 43\% of on-reserve individuals between the ages of 25 and 64, $26.2 \%$ of off-reserve Indians and 11.6 $\%$ of non-aboriginals in BC had had no certificate, diploma or degree (BCStats, 2006). In Canada over 130 Indian Residential Schools were operated from the late 1870's up until 1996 when the last school was closed (Truth and Reconciliation Commission of Canada, TRC, 2013). These schools were funded by the government and run by churches and the goal was to "take the Indian out of the child” (AANDC, 2008). By removing the children by force they were to be isolated from their families and their engagement in their development with the goal of assimilating them into the dominant culture. More than 150000 aboriginal children were taken and approximately 80000 former students, often referred to as survivors as many children died in care, are still alive and eligible for compensation (TRC, 2013). Many young aboriginal people are the first or second generation not to be taken away. In 2005 the First Nations Implementation Plan was established in order to, during a ten year period, address 
This is an Accepted Manuscript of an article published in Disability \& Society on 4 July 2014, available online: http://www.tandfonline.com/10.1080/09687599.2014.923750.

Anne Lindblom, Department of Educational Studies, Karlstad University, Sweden. School of Educational Sciences and Psychology, University of Eastern Finland, Joensuu, Finland.

serious issues that contribute to poverty among aboriginal populations in Canada (AANDC, 2013b).

Seen in the light of the complex history and reality of life as a First Nations person in BC, it would seem that the issue of autism is not urgent or of high priority. For the individual child however it may make a difference in all aspects of life. The purpose of this paper is to review publications regarding autism and aboriginal populations in Canada and other countries is to examine possible explanations for the under-detection.

\section{Methods}

Using the keywords prevalence, aboriginal, culture, ethnicity, indigenous and British Columbia Canada in combination with autism, international databases were searched to find relevant peer-reviewed publications. None of the publications included First Nations children in BC diagnosed with autism and only one paper about First Nations and autism was found (Kapp, 2011). Relevant, current documents on aboriginal affairs discussed at the First Nations Education Steering Committee, FNESC (2012) conference in Vancouver, Canada in November 2012 and an article on Fetal Alchohol Syndrome prevalence among aboriginal peoples were also included for perspective and to put the discussion in context. FNESC was founded in 1992 and works with improving education for First Nations students in BC Canada. All publications are in English, which excludes possible relevant articles in other languages. A total of 16 articles and a doctoral thesis from 2003-2013 from Canada, The United States of America, Australia, The Netherlands, Korea and Ghana were chosen for this review as they were the only publications addressing autism in aboriginal, ethnic or cultural context.

\section{Results}

In the results autism prevalence is discussed first and then possible reasons for underdetection of autism among aboriginal populations as described in the publication review are presented. 
This is an Accepted Manuscript of an article published in Disability \& Society on 4 July 2014, available online: http://www.tandfonline.com/10.1080/09687599.2014.923750.

Anne Lindblom, Department of Educational Studies, Karlstad University, Sweden. School of Educational Sciences and Psychology, University of Eastern Finland, Joensuu, Finland.

\subsection{Prevalence}

Matson and Kozlowski (2011) point out that previous and current studies are not comparable due to changes in diagnostic criteria over the years. Furthermore they suggest that the increase of ASD awareness has influenced the increase in prevalence. The Center for Disease Control and Prevention (Biao, 2012) reported the prevalence rate of ASD in the United States is 1 in 88 in a review of data from fourteen sites between 2000- 2008. American Indian/Alaska Native was one of the populations in which prevalence results were to be reported. However in the results this population is unreported due to small numerators and denominators which produces very unreliable prevalence estimates (J. Biao, personal communication, January 29, 2013). The National Epidemiologic Database for the Study of Autism in Canada (NEDSAC, 2012) reported increased prevalence of autism in four provinces. The agencies providing information to NEDSAC mailed information and consentforms to the parents or legal guardians of the children with autism and those who returned consentforms were interviewed by telephone. Among questions asked were the child's ethnocultural identity but this factor is not defined in the results.

Byrstyn, Sithole and Zwaigenbaum (2010) examined provincial delivery records in Alberta, Canada, from 1998-2004 and ASD follow-ups found in physician billings until March 31, 2008 to determine if there are maternal characteristics associated with increased risk of ASDs in children. They found lower rates of ASDs among children of aboriginal mothers and later diagnosis of these children. A Canadian study on the prevalence of pervasive developmental disorders, which includes ASDs, in Manitoba and Prince Edward Island, PEI, noted significant underrepresentation of autism among aboriginal children (Ouellette-Kuntz et al., 2006). The study included children under the age of 15 years in 2002 identified by workers at Children’s Special services, Manitoba, and by the PEI provincial early intervention coordinator and special education autism coordinator.

\subsection{Diagnostic substitution and symptom expression}

Coo et al. (2008) examined data from 1996 to 2004 about special education classification of children in BC Canada in order to see if children had switched codes to autism from another special education classification, or vice versa, diagnostic substitution. Only one special needs category code can be assigned to a student per year (British Columbia Ministry of Education, 
This is an Accepted Manuscript of an article published in Disability \& Society on 4 July 2014, available online: http://www.tandfonline.com/10.1080/09687599.2014.923750.

Anne Lindblom, Department of Educational Studies, Karlstad University, Sweden. School of Educational Sciences and Psychology, University of Eastern Finland, Joensuu, Finland.

2011) and according to Coo et al. (2008) funding influences which code is assigned. Children with multiple diagnoses may therefore be assigned different special needs codes over time. During the studied timespan the children with an autism special needs classification code increased and Coo et al. (2008) attribute 51.9\% of the increase to diagnostic substitution. Aboriginal students are not specified in the data or results.

Roy and Balaratnasingam (2010) argue that adult indigenous Australian psychiatric patients have been diagnosed with schizophrenia due to autism symptoms of deficits in social interactions and stereotyped interests masking or presenting themselves as a psychotic episode. Of 215 patients diagnosed with schizophrenia, 14 were selected for an evaluation from a psychiatrist specializing in intellectual ability and the developmental history of the patients were taken into consideration. 13 of these 14 patients were diagnosed with ASD and intellectual disability after the assessments from a developmental perspective.

\subsection{Ethnicity and culture}

According to a study on maternal characteristics and obstetric complications, differences in genetic vulnerability within populations can be an explanation for low rates of ASDs among aboriginal children in Alberta, Canada (Byrstyn et al., 2010). Ouellette-Kuntz et al. (2006), Bernier, Mao and Yen (2010) and Ticani, Travers, and Boutot (2010) mention that parents of aboriginal children in Canada and the USA might not be seeking help as a factor leading to underdiagnosis of ASDs within that group. Diagnostic and assessment tools currently utilized lack cultural sensitivity which needs to be acknowledged by researchers and clinicians (Ouelette-Kuntz et al., 2006; Bernier et al., 2010). In a review of literature about cultural influences on decisions regarding treatment for children with ASDs Mandell and Novak (2005) and Bernier et al. (2010) suggest that culture can affect the presentation of ASD as a result of genetic or environmental factors.

Roy and Balaratnasingam (2010) suggest that cultural and language barriers may be a factor in the underdiagnosis of autism in the indigenous population in Australia as well as difficulties in assessing individual developmental history. They also stress that the context of culture should be taken in consideration during the process of diagnosis. Begeer, Bouk Boussaid, Terwogt and Koot (2009) suggest that further research is needed to see if 
This is an Accepted Manuscript of an article published in Disability \& Society on 4 July 2014, available online: http://www.tandfonline.com/10.1080/09687599.2014.923750.

Anne Lindblom, Department of Educational Studies, Karlstad University, Sweden. School of Educational Sciences and Psychology, University of Eastern Finland, Joensuu, Finland.

pediatricians in the Netherlands have stereotypical views that autism is a western disorder. Kim (2012) discusses that cultural factors may be the cause of autism not being well known in Nicaragua. Anthony (2009) notes difficulty in finding publications on autism in Ghana and mentions that most research is done in more developed countries. Though many of the expressions of the symptoms are the same as in other cultures, the study in Ghana revealed certain differences such as the children displaying less repetitive behaviors (Anthony, 2009).

In Australia Leonard et al. (2011) found that aboriginal mothers are less likely to have a child with ASD than a caucasian mother and that due to the complexity of the diagnosing process of ASD that aboriginal children may more readily be diagnosed with intellectual disability, ID. Australian researchers Roy and Balaratnasingam (2010) emphasize that etiological factors are important in diagnosing ASDs and mention Fetal Alcohol Syndrome, FAS, as an important factor due to high alcohol use among indigenous people and stress the need for structure to accurately assess autism within the indigenous population. In the Netherlands Begeer et al. (2009) found that children from non-European minorities, in this case Moroccan or Turkish, were less often diagnosed with autism than Dutch children and conclude that using structured ratings could reduce ethnic bias. They also found that when pediatricians explicitly were asked for ratings of probable ASD in their vignette study, the effect of the child's ethnic background disappeared. Professionals may refrain from further developmental assessment of children in some ethnic groups if they have intellectual disability (Mandell et al., 2009). Bernier et al. (2010) stress the need for clinicians to consider their own cultural biases on the characterization and treatment of autism.

According to Kapp (2011) the Navajo nation has a wellness philosophy that is beneficial for full inclusion and therefore has many qualities that are autism friendly. Viewing a person with autism from a Western society’s perspective, they have a disease, whereas the Navajo culture has acceptance for diversity. The belief that the Creator pre-determines the identities of people helps the Navajo, with and without autism, with their self-worth and the parenting style of being a positive role model instead of disciplining suits the learning styles of people with autism. Furthermore Kapp (2011) notes that society has discriminated and disabled both Navajo and other aboriginal people, and individuals with autism, raising the issues of condescending stereotyping of both groups and forcing aboriginal children to boarding 
This is an Accepted Manuscript of an article published in Disability \& Society on 4 July 2014, available online: http://www.tandfonline.com/10.1080/09687599.2014.923750.

Anne Lindblom, Department of Educational Studies, Karlstad University, Sweden. School of Educational Sciences and Psychology, University of Eastern Finland, Joensuu, Finland.

schools and segregating children with autism to special educational settings. Tincani et al. (2009) and Bernier et al. (2010) state that labels such as autism or other disabilities may not exist in certain cultures. In Nicaragua it appears as autism is not acknowledged and that people are considered the same and embraced in society regardless of disabilities (Kim,2012). Some expressions of autism such as avoiding eye contact may not be problematic in Ghana (Anthony, 2009). In Korea and Ghana the diagnosis of autism is a stigma and shameful for the mother according to Kim (2012) and Anthony (2009).

\subsection{Area of residence}

Burstyn et al. (2010) and Leonard et al. (2011) mention poor access to diagnostic and treatment facilities for aboriginal children living in remote areas of Canada and Australia as a possible explanation for the lower rates of ASDs. Tincani et al. (2009) suggest that geographical location and poor access to health care may result in ethnical minorities being underdiagnosed with ASDs in the USA. Approximately 51000 First Nations people live on reserve and many reserves are in remote areas of BC (BCStats, 2006).

\subsection{Historical oppression and discrimination}

Tincani et al. (2009) mention the impact of historical oppression of aboriginal people contributing to delayed diagnosis of autism among this population. In Australia they have a funding program for children with autism but Wilson and Watson (2011) raise the idea that historical implications of colonialism may discourage the aboriginal community from seeking funding from government interventions. In Canada the reminder of the impact of Indian Residential Schools is currently present as the Truth and Reconciliation Commission is gathering statements from former students (TRC, 2013). In these schools many First Nations children were sexually, physically and emotionally abused. Many children died from the neglect. For over one hundred years First Nations families were separated by force which highly impacts the lives of both survivors of Indian Residential Schools, but also their children and grandchildren (AANDC, 2008).

\section{Figure 1.}

Selected publications for review in chronological order 
This is an Accepted Manuscript of an article published in Disability \& Society on 4 July 2014, available online: http://www.tandfonline.com/10.1080/09687599.2014.923750.

Anne Lindblom, Department of Educational Studies, Karlstad University, Sweden. School of Educational Sciences and Psychology, University of Eastern Finland, Joensuu, Finland.

\section{Autism funding sources and special needs categories in BC, Canada}

In BC there is funding available for both families and schools that is dependent on the child being diagnosed with autism

\subsection{Autism funding programs}

The British Columbia Ministry of Children and Family Development (2013) offers two autism funding programs to enable families to purchase intervention services. These services are intended to assist in communication and social-emotional development along with preacademic or academic skills and life skill competency. The first program offers a substantial amount of funding up until the child turns 6 years. Between the age of 6 and 18 the sum decreases for out of school autism intervention services.

\section{2 special needs categories}

In BC the Ministry of Education has established ten special needs categories to assist school districts in identifying students' needs and providing adequate learning supports and programs (British Columbia Ministry of Education, 2011). Autism spectrum disorder is one of these categories and includes Autistic Disorder, PDD-NOS/Atypical Autism, Asperger

Disorder/Syndrome, Rett Syndrome and Childhood Disintegrative Disorder. There are certain conditions that must be met in order for the school to be eligible for the additional funding. There are three levels of categories and autism spectrum disorders fall under level 2. In addition to this, there may be eligibility for funding for Aboriginal Education or English as a second language.

\section{Discussion}

Though the prevalence of autism has risen, the aboriginal population in BC, Canada is not represented in research publications. Administrative special needs codes in the BC school system and the fact that only one code can be used (Coo et al., 2008) may explain changes in autism prevalence but does not bring any clarity to the underrepresentation of aboriginal children. Research results from international publications offer possible explanations. Autism may present itself in such a manner that it is mistaken for something else, such as schizophrenia (Roy \& Balaratnasingam, 2010), but the presentation of autism can also be 
This is an Accepted Manuscript of an article published in Disability \& Society on 4 July 2014, available online: http://www.tandfonline.com/10.1080/09687599.2014.923750.

Anne Lindblom, Department of Educational Studies, Karlstad University, Sweden. School of Educational Sciences and Psychology, University of Eastern Finland, Joensuu, Finland.

affected by culture (Mandell \& Novak, 2005). Publications indicate that ethnic and cultural factors such as possible differences in genetic vulnerability (Byrstyn et al., 2010) and how parents and others view the ways autism presents itself (Kapp, 2011) can be possible reasons for the underrepresentation of autism among aboriginal populations in BC. Cultural context in usually not taken into consideration in the diagnosis process (Ouelette-Kuntz et al., 2006). Along with contextual cultural aspects there is need for structure in the diagnosis procedure (Begeer et al., 2009). In Australia culture and language barriers are considered a possible factor in the underdiagnosis of autism within the aboriginal population (Roy \& Balaratnasingam, 2010), which may also be the case in BC. Living in remote areas with poor access to diagnostic and treatment facilities is mentioned in publications from Canada and Australia (Burstyn et al., 2010; Leonard et al., 2011).

Begeer et al. (2009) and Kapp (2011) mention stereotyping which is an issue for all indigenous peoples who were colonized. Ethnic bias in autism referral was found in Australia where aboriginal children were more likely to be diagnosed with intellectual disability (Leonard et al., 2011; Roy \& Balaratnasingam, 2010). In some ethnic groups it appears that professionals do not further investigate developmental aspects if the child has intellectual disability (Mandell et al., 2009). FAS, is mentioned as a cause of autism and the need to explore etiology is pointed out as essential (Roy \& Balaratnasingam, 2010). According to heretohelp (2013) which is a project within the BC Partners for Mental Health and Addictions Information, aboriginal mothers are more likely to have children born with FAS and other problems due to higher rates of substance use during pregnancy than other women. However a Canadian review of literature on FAS prevalence among aboriginal people states that results are inconsistent and though certain studies report higher prevalence among this group, more research has to be done (National Collaborating Centre for Aboriginal Health, 2009). Perhaps referral bias or stereotypical views on the aboriginal population among professionals lie behind symptoms of autism being overlooked or being recognized as FAS. Stereotyping and residential schools (TRC, 2008) are currently hot topics within the aboriginal community in BC, especially since the Statement of Apology (AANDC, 2008) from the Prime Minister of Canada, and the concern of discrimination is ever-present in everyday life situations. 
This is an Accepted Manuscript of an article published in Disability \& Society on 4 July 2014, available online: http://www.tandfonline.com/10.1080/09687599.2014.923750.

Anne Lindblom, Department of Educational Studies, Karlstad University, Sweden. School of Educational Sciences and Psychology, University of Eastern Finland, Joensuu, Finland.

\section{Conclusions}

Despite efforts to include indigenous populations in national surveys for autism in Canada and the USA, they are not visible in the presented results. Further research is needed to explain why First Nations children in BC with autism are not represented in research publications in order to influence practice and policy. Perhaps administrative data does not adequately present this group and other research methods need to be utilized to make them more apparent. Correct diagnosis and use of culturally sensitive tools for diagnosis and assessment is crucial so adequate treatment, interventions, education and eligibility for funding is made available to the aboriginal population. If there are protective genetic, ethnic or cultural factors against autism within the aboriginal population, it could be beneficial and interesting globally to identify these factors. It is essential that barriers of ethnic bias, discrimination and the impact of historical oppression are eliminated to ensure that First Nations and other aboriginal children with autism have the possibilities to reach their full potential and that their rights are recognized and respected. However, there is a risk that an autism diagnosis becomes just another label for an already categorized and stigmatized First Nations child. Therefore it is crucial that the process of diagnosis, policy development and research are conducted in collaboration with the First Nations people.

\section{References}

Aboriginal Affairs and Northern Development Canada. AANDC. (2012a). Aboriginal peoples and communities. Retrieved from http://www.aadnc-aandc.gc.ca/eng/1100100013785/1304467449155

Aboriginal Affairs and Northern Development Canada, AANDC (2013a). First Nations. Retrieved from http://www.aadnc-aandc.gc.ca/eng/1100100013791/1100100013795

Aboriginal Affairs and Northern Development Canada, AANDC (2013b). First Nations Implementation Plan. Retrieved from http://www.aadnc-aandc.gc.ca/eng/1100100013797/1100100013798

Aborinal Affairs and Northern Development Canada. AANDC. (2012c). Indian Status. Retrieved from http://www.aadnc- aandc.gc.ca/eng/1100100032374/1100100032378

Aboriginal Affairs and Northern Development Canada. AANDC. (2008). Statement of Apology Retrieved from http://www.aadnc-aandc.gc.ca/eng/1100100015644/1100100015649

Aboriginal Affairs and Northern Development Canada. AANDC. (2012b). Terminology. Retrieved from http://www.aadnc-aandc.gc.ca/eng/1100100014642/1100100014643

Anthony, J.H. (2009). Towards inclusion: Influences of Culture and Internationalisation on Personhood, Educational Access, Policy and Provision for Students with Autism in Ghana (Doctoral dissertation, University of Sussex). Retrieved from http://eprints.sussex.ad.uk/

BCStats. (2006). Census 2006. Aboriginal profiles. Aboriginal compared to the non-aboriginal population. 
This is an Accepted Manuscript of an article published in Disability \& Society on 4 July 2014, available online: http://www.tandfonline.com/10.1080/09687599.2014.923750.

Anne Lindblom, Department of Educational Studies, Karlstad University, Sweden. School of Educational Sciences and Psychology, University of Eastern Finland, Joensuu, Finland.

Retrieved from

http://www.bcstats.gov.bc.ca/StatisticsBySubject/AboriginalPeoples/CensusProfiles/2006Census.aspx

Beeger, S., Bouk, S.E., Boussaid, W., Terwogt, M.M. \& Koot, H.M. (2009). Underdiagnosis and Referal Bias of Autism in Ethnic Minorities. Journal of Autism and Developmental Disorders, 39(1), 142-148.

Bernier, R., Mao, A., Yen, J. (2010). Psychopathology, Families and Culture: Autism. Child and adolescent psychiatric clinics of North America, 19(4), 855-867.

Biao, J.\& Centers for Disease Control and Prevention,(2012). Prevalence of Autism Spectrum Disorders: Autism and Developmetal Disabilities Monitoring Network, 14 Sites, United States, 2008. Morbidity and Mortality Weekly Report. Surveillance summaries. Centers For Disease Control And Prevention, 61(3)

British Columbia Ministry of Education. (2011). Special Education Services: A Manual of Policies, Procedures and Guidelines - March 2011. Retrieved from: http://www.bced.gov.bc.ca/specialed/ppandg.htm

British Columbia Ministry of Children and Family Development. (2013). Eligibility for Autism Programs Policy. Retrieved from http://www.mcf.gov.bc.ca/spec_needs/pdf/eligibility_autism.pdf

Burstyn, I., Sithole, F. \& Zwaigenbaum, L. (2010). Autism spectrum disorders, maternal characteristics and obstetric complications among singletons born in Alberta, Canada. Chronic Diseases in Canada, 30(4), 125134.

Coo, H.,Ouellette-Kuntz, H., Lloyd, J.E.V., Kasmara, L., Holden, J.J.A. \& Lewis, M.E.S. (2008). Trends in autism prevalence: Diagnostic substitution revisited. Journal of autism and Developmental Disorders, 38(4), 1036- 1046.

First Nations Education Steering Committee. FNESC. (2012). Retrieved from http://www.fnesc.ca

Foucault, M. (1988). Madness and civilization. A history of insanity in the age of reason. New York: Vintage.

Government of Canada. (1985/2011). Indian Act. Retrieved from http://laws-lois.justice.gc.ca/eng/acts/I-5/

heretohelp. (2013).Retrieved from http://heretohelp.bc.ca/factsheet/aboriginal-mental-health-and-substance-use

Hyman, S.L. (2013). New DSM-5 includes changes to autism criteria. AAP News, doi:

10.1542/aapnews.20130604-1

Kapp, S.K. (2011). Navajo and autism: the beauty of harmony. Disability and Society, 6(5), 583-595.

Kim, H. U. (2012). Autism across cultures: rethinking autism. Disability and Society, 27(4), 535-545.

Leonard, H., Glasson, E., Nassar, N. Whitehouse, A., Bebbington, A. Bourke, J., Jacoby, P., Dixon, G., Malacova, E., Bower, C. \& Stanley, F. (2011). Autism and Intellectual Disability Are Differentially Related to Sociodemographic Background at Birth. PloS ONE, 6(3), 1-9.

Mandell, D.S. \& Novak, M. (2005). The Role of Culture in Families’ Treatment Decisions for Children with Autism Spectrum Disorders. Mental Retardation and developmental disabilities research reviews, 11(2), 110115.

Mandell, D.S., Wiggins, L.D, Arnstein Carpenter, L., Daniels, J., DiGuiseppi, C., Durkin, M.S., Giarelli, E., Morrier, M. J., Nicholas, J.S., Pinto-Martin, J.A., Shattuck, P.T., Thomas, K.C., Yergin-Allsopp, M. Kirby, R.S. (2009). Racial/Ethnic Disparities in the Identification of Children With Autism Spectrum Disorders. American Journal of Public Health, 99(3), 493-498.

Matson, J. L.\& Kozlowski, A. M. (2011). The increasing prevalence of autism spectrum disorders. Research in Autism Spectrum Disorders, 5(1), 418-425. 
This is an Accepted Manuscript of an article published in Disability \& Society on 4 July 2014, available online: http://www.tandfonline.com/10.1080/09687599.2014.923750.

Anne Lindblom, Department of Educational Studies, Karlstad University, Sweden. School of Educational Sciences and Psychology, University of Eastern Finland, Joensuu, Finland.

National Collaborating Centre for Aboriginal Health. (2009). Fetal Alcohol Syndrome \& Fetal Alcohol Spectrum Disorder Among Aboriginal Peoples. A review of prevalence. Retrieved from http://www.nccahccnsa.ca/docs/child\%20and\%20youth/NCCAH-paper-FASD-aboriginalprevalencereview-sept2009webready.pdf

National Epidemiologic Database for the Study of Autism in Canada. NEDSAC. (2012). Changes in the Prevalence of Autism Spectrum Disorders in Newfoundland and Labrador, Prince Edward Island, and Southeastern Ontario. Findings from the National Epidemiologic Database for the Study of Autism in Canada (NEDSAC)March 2012.Retrieved from

http://authoring.wp.queensu.ca/lenya/nedwww/live/Publications/FamilyUpdates/NEDSAC_Report_March2012. pdf

Ouellette-Kuntz, H., Coo, H., Yu, C.T., Chudley, A.E., Noonan, A., Breitenbach., Ramji, N., Prosick, T. Bedard, A. \& Holden, J.J.A. (2006). Prevalence of Pervasive Developmental Disorders in Two Canadian Provinces. Journal of Policy and Practice in Intellectual disabilities, 3(3), 164-172.

Roy, M. \& Balaratnasingam, S. (2010). Missed diagnosis of autism in an Australian Indigenous psychiatric population. Australasian Psychiatry, 18(6), 534-537. doi:10.3109/10398562.2010.498048

Stienstra, D. (2012). Race/ethnicity and disability studies. Towards an explicitly intersectional approach. In N. Watson, A. Roulstone \& C.Thomas (Eds.), Routledge Handbook of Disability Studies (pp.376-389). New York, NY: Routledge.

Szasz, T.S.(1974). The myth of mental illness. Foundations of a theory of personal conduct. New York: Harper \& Row.

Tincani, M., Travers, J. \& Boutot, A. (2009). Race, Culture, and Autism Spectrum Disorder: Understanding the Role of Diversity in Successful Educational Interventions. Research \& Practice for Persons with Severe Disabilities, 34(3-4) 81-90.

Truth and Reconciliation Commission of Canada. TRC, (2013). Retrieved from http://www.trc.ca/websites/trcinstitution/index.php?p=3

Wilson, K. \& Watson, L. (2011). Autism Spectrum Disorder in Australian Indigenous Families: Issues of Diagnosis, Support and Funding. Aboriginal \& Islander Health Worker Journal, September/October 2011, 35(5), 17-18. 